\title{
Two-Body Relationship Between the Pearson-Takai-Halicioglu-Tiller and the Biswas-Hamann Potential Functions
}

\author{
Teik-Cheng Lim \\ Nanoscience and Nanotechnology Initiative, Faculty of Engineering, \\ 9 Engineering Drive 1, National University of Singapore, S 117576, Republic of Singapore \\ Received on 6 May, 2005
}

\begin{abstract}
An approximate and a good parametric relationship between the Pearson-Takai-Halicioglu-Tiller (PTHT) and the Biswas-Hamann (BH) empirical potential energy functions is developed for the case of 2-body interaction. The approximate relationship between PTHT and BH was obtained by equating the zeroth up to the second order of the potential functions' derivative with respect to the interatomic distance at the equilibrium bond length, followed by comparison of coefficients at the repulsive and attractive terms. A refined relationship was then suggested by including the third order derivative. Plots of non-dimensional 2-body energy versus the non-dimensional interatomic distance verified the analytical relationships developed herein. Finally, the physical significance of the developed parametric relationships is discussed with reference to conservative design methodology.
\end{abstract}

\section{INTRODUCTION}

Parametric relationships between various interatomic potential energy function of the same category of $n$-body interaction can be useful when available physical data and computational softwares are based on parameters of different potential functions. In addition to providing a way for converting potential functions, any discrepancy between these potential functions will provide insight on how the choice of potential functions will affect the outcome of simulation results. Mathematical relationships for relating molecular potential functions used in chemical computation have been developed [1-7]. These relationships were improved and subsequently developed into a molecular potential function converter software [8]. However, parametric relationships between potential energy functions used for computational condensed matter properties are lacking. Though Stoneham et al. [9] performed a comparison of eight potential functions for silicon, the functions considered are under the category of valence-force potentials which are useful only for describing small distortions from equilibrium. A set of six potential functions were compared by Balamane et al. [10] but the potential functions plotted were based on parameters obtained via experimental curve-fitting instead of analytical conversion of parameters. In this paper, a set of analytical relationship is developed for converting parameters between the 2-body interactions in the Pearson-Takai-Halicioglu-Tiller (PTHT) and the Biswas-Hamann (BH) potential functions. The PTHT potential function was initially developed for silicon [11], whereby its 2-body energy is taken from the Lennard-Jones potential [12]. The terminology selected here is the "2-body portion of PTHT" instead of "Lennard-Jones" to conform to previous work [13,14], whereby the phrases "2-body portion of Murrell-Mottram" (MM) [15] and the "2-body portion of Bauer-Maysenholder-Seeger" (BMS) [16] were adopted instead of the original terms "Rydberg" [17] and "Buckingham" [18] potentials respectively, due to the emphasis for application in many-body potentials used in condensed matter computation [19]. See also Fig.1 whereby the parametric connections amongst the 2-body potential functions of PTHT [11],
MM [15], BMS [16], Kaxiras-Pandey (KP) [20] and BH [21] were connected.

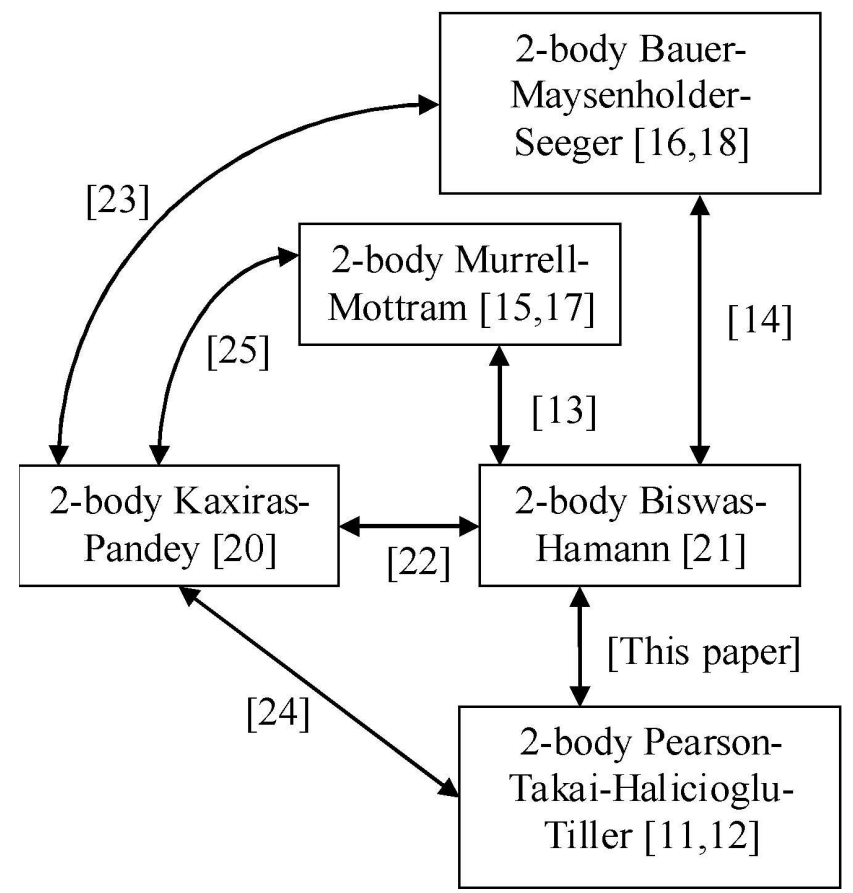

FIG. 1: Recently established relationships and presently developed relationship.

\section{ANALYSIS}

As with PTHT, the BH potential function was also developed for silicon [21]. Similar to PTHT, BH potential function belongs to a broad category of potential functions whereby the total energy of a system is a summation of all 2-body and all 3-body interactions 


$$
\Phi=\phi_{2-b o d y}+\phi_{3-b o d y}=\sum_{i<j} U_{i j}+\sum_{i<j<k} W_{i j k} .
$$

In addition, the 2-body interactions in PTHT and BH belong to a sub-category of potential function which consist of two distinct parts: a repulsive term and an attractive term,

$$
U_{i j}=U_{i j}^{\text {repul }}+U_{i j}^{\text {attract }} .
$$

Specifically, the PTHT and BH potential functions for 2body interactions are written as

$$
U_{P T H T}=\varepsilon\left[\left(\frac{R}{r}\right)^{12}-2\left(\frac{R}{r}\right)^{6}\right]
$$

and

$$
U_{B H}=A_{1} \exp \left(-\lambda_{1} r\right)+A_{2} \exp \left(-\lambda_{2} r\right)
$$

respectively, where $\varepsilon$ is the magnitude of the minimum welldepth, $r$ is the interatomic distance and $R$ is the distance at equilibrium for the case of PTHT whilst $A_{i}$ are the coefficients and $\lambda_{i}$ are the indices of the repulsive $(i=1)$ and attractive $(i=2)$ terms. Furthermore, we note that both the 2-body potentials are such that the functional forms in the repulsive and attractive terms are similar for each potential function, i.e. $\varepsilon(R / r)^{6 i}$ for PTHT and $A_{i} \exp \left(-\lambda_{i} r\right)$ for BH, whereby $i=1,2$. Let

$$
\left(\frac{\partial^{n} U_{B H}}{\partial r^{n}}\right)_{r=R}=\left(\frac{\partial^{n} U_{P T H T}}{\partial r^{n}}\right)_{r=R}
$$

for $n=0,1,2,3$, we have

$$
\left[\begin{array}{ll}
\xi_{1}^{0} & \xi_{2}^{0} \\
\xi_{1}^{1} & \xi_{2}^{1} \\
\xi_{1}^{2} & \xi_{2}^{2} \\
\xi_{1}^{3} & \xi_{2}^{3}
\end{array}\right]\left\{\begin{array}{l}
\exp \left(-\xi_{1}\right) \\
\exp \left(-\xi_{2}\right)
\end{array}\right\}=\varepsilon\left\{\begin{array}{c}
-1 \\
0 \\
72 \\
1512
\end{array}\right\}
$$

where

$$
\xi_{i}=\lambda_{i} R \quad ; \quad(i=1,2)
$$

are the scaling factors. Eliminating the terms $A_{i} \exp \left(-\xi_{i}\right)$ for $i=1,2$ from Eq.(6), we arrive at the upper and lower solutions of one scaling factor in terms of another scaling factor,

$$
\left\{\begin{array}{c}
\xi_{i}^{+} \\
\xi_{i}^{-}
\end{array}\right\}=\left\{\begin{array}{c}
\xi_{j} \\
\left(72 / \xi_{j}\right)
\end{array}\right\}
$$

Solving the first two rows of Eq.(6) simultaneously,

$$
A_{i}=\varepsilon\left(\frac{\xi_{j}}{\xi_{i}-\xi_{j}}\right) \exp \left(\xi_{i}\right)
$$

Equation (9) enables the 2-body BH potential, i.e. Eq.(4), to be rewritten in a loose form

$$
\begin{gathered}
U_{B H}=\varepsilon\left[\left(\frac{\xi_{2}}{\xi_{1}-\xi_{2}}\right) \exp \left(\xi_{1}\left(1-\frac{r}{R}\right)\right)-\right. \\
\left.\left(\frac{\xi_{1}}{\xi_{1}-\xi_{2}}\right) \exp \left(\xi_{2}\left(1-\frac{r}{R}\right)\right)\right],
\end{gathered}
$$

thereby signifying that the upper solution of Eq.(8) to be invalid. An approximate relation between PTHT and BH can be obtained by comparing the coefficients of Eq.(10) with those of Eq.(3) to give

$$
\xi_{1}=2 \xi_{2}
$$

Substituting Eq.(11) into the lower solution in Eq.(8), we have $\xi_{1}=12$ and $\xi_{2}=6$. For attaining better accuracy, we eliminate the terms $A_{i} \exp \left(-\xi_{i}\right)$ for $i=1,2$ by solving the last three rows of Eq.(6) to give

$$
\xi_{1}+\xi_{2}=21
$$

From Eq.(12) and the lower solution of Eq.(8), we obtain

$$
\left\{\begin{array}{l}
\xi_{1} \\
\xi_{2}
\end{array}\right\}=\frac{1}{2}\left\{\begin{array}{l}
21+\sqrt{153} \\
21-\sqrt{153}
\end{array}\right\} .
$$

\section{RESULTS AND DISCUSSION}

A summary of 2-body interaction relationship between the parameters of PTHT and BH is furnished in Table 1. For verification, graphs of non-dimensional 2-body energy $(U / \varepsilon)$ were plotted against the non-dimensional bond length $(r / R)$. Figure 2(a) depicts the approximate and improved BH curves based on PTHT parameters. A close-up view at the minimum well depth is shown in Fig. 2(b). For both illustrations, the developed approximate and a good relationship between the 2-body energy of the PTHT and the BH potential functions has been shown to be valid. It is concluded herein that by considering higher derivative ( $3^{\text {rd }}$ order), we obtain a much improved matching in comparison to the consideration of lower derivative ( $2^{\text {nd }}$ order).

In addition to gaining insight on the connection between these potential functions, the parametric relationships also enable the discrepancies to be clearly observed [26], thereby paving a way for the conservative design methodology to be developed. To further appreciate the physical implications of the relationships furnished in Table 1, we introduce the nondimensionalized interatomic force as

$$
F^{*}=-\frac{F R}{\varepsilon}
$$


Table 1. Summary of 2-body relationship between PTHT and BH potential function parameters.

\begin{tabular}{|c|c|c|}
\hline $\begin{array}{lll}\begin{array}{l}\text { Potential functions } \\
\text { parameters }\end{array} & \text { and }\end{array}$ & Parametric relationships & Scaling Factors \\
\hline $\begin{array}{l}U_{P T H T}= \\
U_{P T H T}(\varepsilon, R, r) \\
U_{B H}= \\
U_{B H}\left(A_{1}, A_{2}, \lambda_{1}, \lambda_{2}\right)\end{array}$ & $\begin{array}{l}A_{1}=\varepsilon\left(\frac{\xi_{2}}{\xi_{1}-\xi_{2}}\right) \exp \left(\xi_{1}\right) \\
A_{2}=-\varepsilon\left(\frac{\xi_{1}}{\xi_{1}-\xi_{2}}\right) \exp \left(\xi_{2}\right) \\
\lambda_{1}=\frac{\xi_{1}}{R} \\
\lambda_{2}=\frac{\xi_{2}}{R}\end{array}$ & $\begin{array}{l}\text { Approximate: } \\
\left\{\begin{array}{l}\xi_{1} \\
\xi_{2}\end{array}\right\}=\frac{1}{2}\left\{\begin{array}{l}18+\sqrt{36} \\
18-\sqrt{36}\end{array}\right\}=\left\{\begin{array}{c}12 \\
6\end{array}\right\} \\
\text { Improved: } \\
\left\{\begin{array}{l}\xi_{1} \\
\xi_{2}\end{array}\right\}=\frac{1}{2}\left\{\begin{array}{l}21+\sqrt{153} \\
21-\sqrt{153}\end{array}\right\}=\left\{\begin{array}{c}16.6847 \\
4.3153\end{array}\right\}\end{array}$ \\
\hline
\end{tabular}

where the absolute interatomic force is defined from

$$
F=-\frac{\partial U}{\partial r}
$$

A negative sign is incorporated into Eq.(14) in order to give positive values of the force when the bonded atoms are stretched. Hence

$$
F_{P T H T}^{*}=-12\left[\left(\frac{R}{r}\right)^{13}-\left(\frac{R}{r}\right)^{7}\right]
$$

and

$$
F_{B H}^{*}=-\left[\lambda_{1} A_{1} \exp \left(-\lambda_{1} r\right)+\lambda_{2} A_{2} \exp \left(-\lambda_{2} r\right)\right] \frac{R}{\varepsilon}
$$

Substituting Eqs.(7) and (9) into Eq.(17), we arrive at

$$
F_{B H}^{*}=\frac{\xi_{1} \xi_{2}}{\xi_{2}-\xi_{1}}\left\{\exp \left[\xi_{1}\left(1-\frac{r}{R}\right)\right]-\exp \left[\xi_{2}\left(1-\frac{r}{R}\right)\right]\right\} .
$$

Figure 3 shows the plots of $F_{P T H T}^{*}, F_{B H}^{*}$ (approximated) and $F_{B H}^{*}$ (improved) versus the non-dimensionalized interatomic distance. The approximated and improved $F_{B H}^{*}$ are based on the values of the scaling factors shown in Table 1. The higher peak for $\mathrm{BH}$ (approx) in Fig. 3 corresponds to the higher slope of the BH(approx) inflexion point in Fig. 2(a) in comparison to PTHT. We also note that the occurrence of the peak occurs first for $\mathrm{BH}$ (approx) in Fig. 3 because the inflexion point occurs first for BH(approx) in Fig. 2(a). Prescription of $F^{*} \in[2.69,3.00]$ would give simulation results which predicts bond dissociation based on PTHT but no dissociation based on $\mathrm{BH}$ (approx). However, prescription of $(r / R) \in[1.110,1.115]$ would lead to simulated bond dissociation based on $\mathrm{BH}$ (approx) but no dissociation based on PTHT. Whilst the deviation of BH(approx) from PTHT is about $11.5 \%$ at the peak force, the deviation of $\mathrm{BH}$ (improv) from PTHT is about $0.1 \%$.

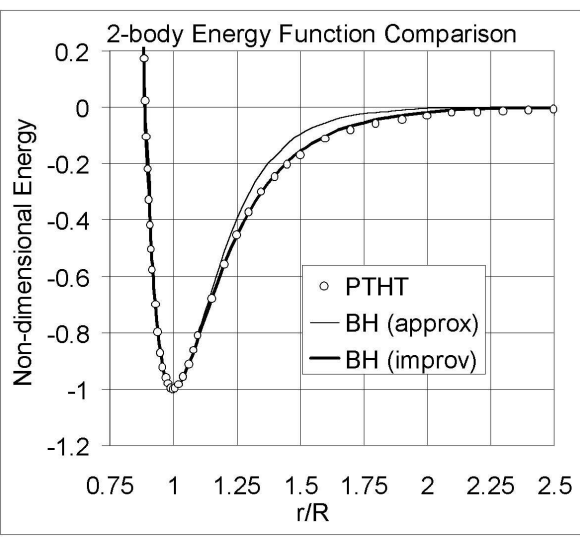

(a)

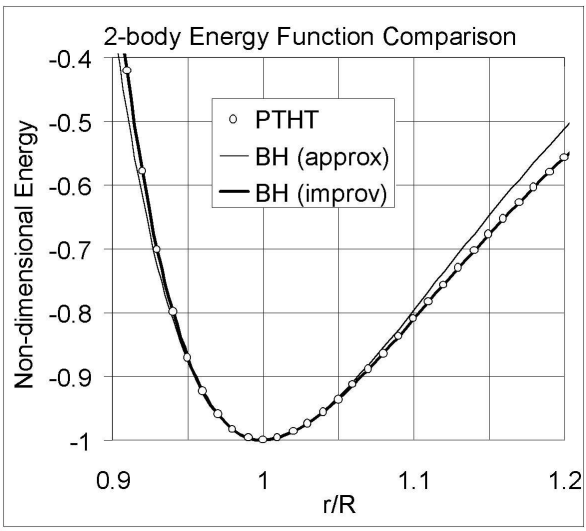

(b)

FIG. 2: Comparison of 2-body energy function according to PTHT and $\mathrm{BH}$ curves (a) long range view, and (b) close up view at minimum well depth.

\section{CONCLUSIONS AND RECOMMENDATION}

With these observations, we conclude the following:

There is a large improvement in the parametric relationship when the order of derivative considered in increased from second order to the third order. With the good agreement between PTHT and BH(improv), there is no practical requirement to incorporate the fourth order due to diminishing returns. 


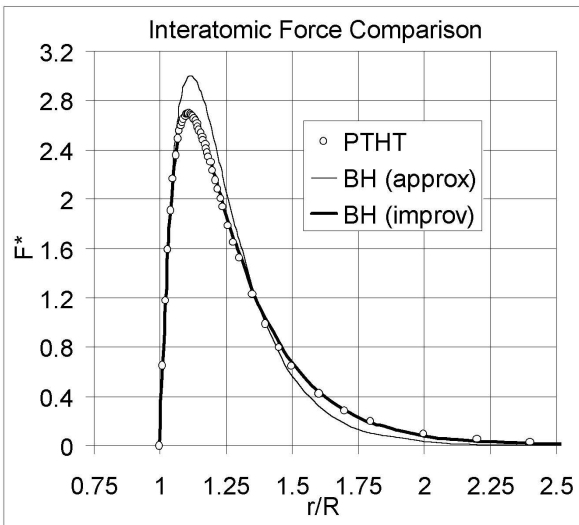

FIG. 3: Comparison of interatomic force of PTHT and BH.
BH potential is more suitable for hard bonds whilst PTHT is more suitable for soft bonds. Note that the bond hardness is different from a strong bond in that the former is a description of the abruptness in the rise of interatomic energy with interatomic distance whilst the latter the magnitude of the minimum well-depth.

It follows that PTHT is advisable when load is prescribed whilst $\mathrm{BH}$ is advisable when distortion is prescribed for conservative design.

In view of the excellent agreement between parameters of the PTHT and $\mathrm{BH}$ (improv) potential functions, a set of relationships between parameters the 3-body energy portion for these two potential functions is suggested for future work.
[1] T.C. Lim, J. Math. Chem. 33, 279 (2003).

[2] T.C. Lim, J. Math. Chem. 34, 221 (2003).

[3] T.C. Lim, J. Math. Chem. 36, 139 (2004).

[4] T.C. Lim, J. Math. Chem. 36, 147 (2004).

[5] T.C. Lim, J. Math. Chem. 36, 261 (2004).

[6] T.C. Lim, Z. Naturforsch. A 58, 615 (2003).

[7] T.C. Lim, MATCH Commun. Math. Comput. Chem. 49, 155 (2003).

[8] T.C. Lim, MATCH Commun. Math. Comput. Chem. 50, 185 (2004).

[9] A.M. Stoneham, V.T.B. Torres, P.M. Masri, and H.R. Schober, Phil. Mag. A 58, 93 (1988).

[10] H. Balamane, T. Halicioglu, and W.A. Tiller, Phys. Rev. B 46, 2250 (1992).

[11] E. Pearson, T. Takai, T. Halicioglu, and W.A. Tiller, J. Cryst. Growth 70, 33 (1984)

[12] J.E. Lennard-Jones, Proc. Roy. Soc. Lond. A 106, 463 (1924).
[13] T.C. Lim, Z. Naturforsch. A 59, 116 (2004).

[14] T.C. Lim, Czech. J. Phys. 54, 553 (2004).

[15] J.N. Murrell and R.E. Mottram, Mol. Phys. 69, 571 (1990).

[16] R. Bauer, W. Maysenholder, and A. Seeger, Phys. Lett. A 90 55 (1982)

[17] R. Rydberg, Z. Phys. 73, 376 (1931).

[18] R.A. Buckingham, Proc. Roy. Soc. Lond. A 168, 264 (1938).

[19] S. Erkoc, Phys. Rep. 278, 79 (1997).

[20] E. Kaxiras and K.C. Pandey, Phys. Rev. B 38, 12736 (1988).

[21] R. Biswas and D.R. Hamann, Phys. Rev. Lett. 55, 2001 (1985).

[22] T.C. Lim, Czech. J. Phys. 54, 947 (2004).

[23] T.C. Lim, MATCH Commun. Math. Comput. Chem. 54, 29 (2005).

[24] T.C. Lim, Physica Scripta 70, 347 (2004).

[25] T.C. Lim, Chin. J. Phys. 43, 43 (2005).

[26] T.C. Lim, Chin. Phys. Lett. 21, 2167 (2004). 